\title{
Comparison of User Experiences of Augmented Reality-based Car Maintenance Contents Using Mobile Devices and Hololens
}

\author{
Myeong-Hyeon Heo1), Jaewoon Lee ${ }^{1)}$, Van Thanh Tran ${ }^{1)}$, Eun-Young Lee ${ }^{1)}$, Minsang \\ $\mathrm{Kim}^{1)}$, Dongho Kim²)
}

\begin{abstract}
With the recently developed glass type devices, augmented reality is expected to provide more intuitive interaction and be actively used in work environments leaving the hands free. In addition, since eco-friendly new types of cars were launched in the automobile industry, there has been a shortage of skilled maintenance personnel. In order to solve this problem, using augmented reality technology based on Hololens can make it possible to deliver the exact information in real time in various maintenance situations. Therefore, this study intends to evaluate, compare and analyze the user experiences of augmented reality contents in the field of car maintenance between mobile devices and Hololens and use the results as the materials for creating novel services in the future.
\end{abstract}

Keywords : Augmented Reality, Hololens, Automobile Maintenance, User Experience

\section{Introduction}

Augmented reality has developed to the glass form such as Google Glass or HoloLens from the conventional augmented visualization using desktop computers and mobile devices. The recent form can provide more intuitive interactions and it is expected that both hands will be used freely and actively in various work environments[1][2].

The automobile industry provides diverse functions by combining the advanced technologies and software programs and introduces new types of automobiles such as eco-friendly automobiles. Consequently, the structure and functions of the automobile became more complex and the design and options have been diversified.

Received(October 10, 2017), Review Result(1st: November 02, 2017, 2nd: November 28, 2017), Accepted(December 10, 2017)

${ }^{1}$ Dept. of Information Communication, Materials, and Chemistry Convergence Technology, Soongsil Univ., Sangdo-ro, Dongjak-gu, Seoul, 06978, Korea

email: heomaeng@gsclab.kr (other authors don't want to show their e-mail addresses)

${ }^{2}$ (Corresponding Author) Dept. of Digital Media, Soongsil Univ., Sangdo-ro, Dongjak-gu, Seoul, 06978, Korea email: cg@su.ac.kr

* 이 논문은 2017년도 정부(미래창조과학부)의 재원으로 정보통신기술진흥센터의 지원을 받아 수행된 연구임 (No.2017-0-01785, 첨단융복합기술개발) 
However, the number skilled technicians is limited[3]. This problem can be overcome if the technical document, database, and 3D sensing information are projected to the actual objects in real time in order to accurately deliver the necessary information by using the HoloLens based augmented reality technology to the automobile maintenance situation. It can also reduce the efforts and training required to obtain the skills. Moreover, it can help automobile technicians maintain the automobiles more intuitively and safely. Additionally, manufacturers and automobile maintenance service companies are able to allocate service personnel more efficiently so they can respond to various situations while hiring a smaller number of employees. It will ultimately improve customer satisfaction.

Multiple studies evaluated the interaction between mobile devices and programs implementing augmented reality in the field of education and training. These studies showed the augmented reality technology was a medium increasing the potential and interest in learning[4][5]. However, there are no enough analyses on the user experience of HoloLens, which is remarkably improved augmented reality equipment. Therefore, this study aims to provide a baseline data for developing an effective service in the future by comparing the user experience of augmented reality contents (i.e., mobile devices and HoloLens) in the automobile maintenance field.

\section{Related Studies}

Augmented reality, unlike virtual reality, shows a virtual object superimposed on the real space in order to provide additional information, which is difficult to obtain in the real world[6]. Azuma (1997) explained the realization of augmented reality in three aspects. First, the real world and the virtual world shall be combined and create a seamless implementation. Second, a user and a system need to communicate ceaseless in real time. Third, a virtual object shall be aligned with a real object in the real world accurately three-dimensionally to satisfy the five senses of a user and improve immersion feeling. Azuma argued that augmented reality is not produced just by combining a real world and a virtual world but by making them mutually circulating and having an organic relationship[7].

Billinghurst (2002) defined the five advantages of augmented reality. The first is to provide a smooth interaction in real time by combining a real-world element with a virtual image. The second is to enhance the sense of reality by providing information beyond what is visible by combining the elements of reality in the real world with virtual information, which can be considered another reality. The third is to provide a user with augmented realism and enhanced digital information in the virtual environment merged with a real environment, beyond the spatial constraints. Fourth, it allows the provision of a new metaphor to the interface, such as using surrounding objects as a digital medium, beyond the general operation using the mouse and keyboard, which are conventional input devices of the computer. Fifth, it offers smooth conveyance between the 
real world and the virtual world[8].

Owing to the development of head mounted display(HMD) based technologies, Microsoft recently released HoloLens, which can be used without mobile or desktop devices. HoloLens has brought new possibilities to the field of augmented reality in the aspect that recognizes the characteristics of an environment in addition to save them in the actual space as well as coordinate a 3D model in the real space. Especially, HMD-based augmented reality has the advantage of creating immersive and interactive contents because there are no limitations of screen and activity radius[9][10].

\section{Experimental Methods}

\subsection{Subjects of Experiment}

This study targeted 44 undergraduate students majoring in Automotive and Mechanical Engineering in a college located in Seoul, Korea, who have not used HoloLens before. They were divided into two groups of 22 subjects each. The use of mobile device and that of HoloLens were used as an independent variable and the evaluation score after the use was set as a dependent variable.

\subsection{Experiment Procedure}

[Table 1] Augmented Reality Contents

\begin{tabular}{ccc}
\hline Classification & Content $\mathrm{A}$ & Content B \\
\hline Image & Motion of the inner piston located in the & None \\
\hline Animation & engine miniature & Display the part name by recognizing the \\
\hline Label & None & position
\end{tabular}

Two contents were created as virtual objects by utilizing Unity 3D[11] and Vuforia SDK[12] based on literature review and existing augmented reality application case studies. Content A animated the structure and movement of the inner piston of an engine miniature and Content $\mathrm{B}$ recognized the accurate position of a part and displayed it by using a label [Table 1].

[Table 2] Resolution of Mobile Devices \& Hololens and Performance of Camera 


\begin{tabular}{|c|c|c|}
\hline Model & Samsung Galaxy Note8 & MS $\quad$ Hololens \\
\hline \multicolumn{3}{|l|}{ Image } \\
\hline Resolution & 2960×1440(Quad HD+) & $1268 \times 720(720 p)$ \\
\hline Camera & 12 million pixels & 2.4 million pixels \\
\hline
\end{tabular}

These augmented reality contents were observed by the subjects in a studio equipped with two stand lamps with consideration of the limitations of the existing HoloLens hardware developed so far and for a smooth experimental progress [Fig. 1(b)]. The mobile device user group held the device with one hand and operated the device with the other hand in order to evaluate the user experience [Fig. 1(c)]. The HoloLens user group operated the device with gesture and voice input in order to evaluate the user device experience [Fig. 1(d)].

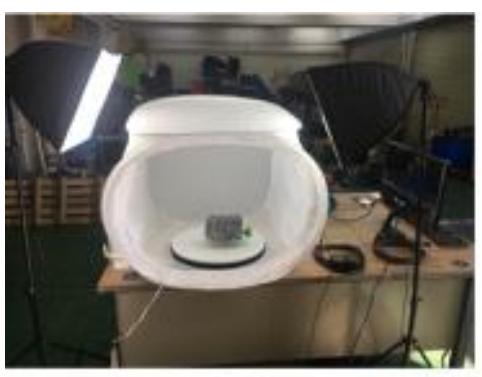

(a)

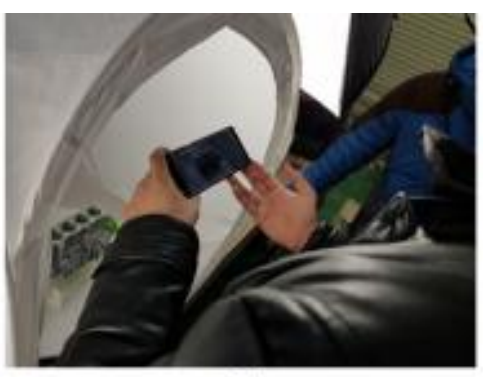

(b)

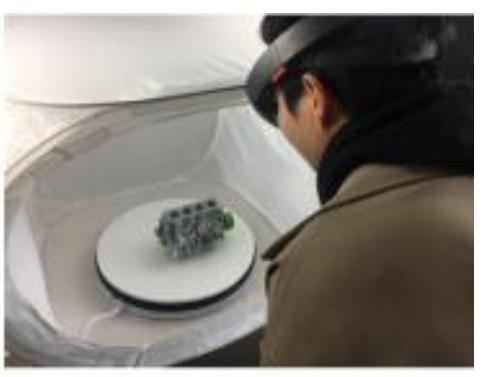

(C)

[Fig. 1] (a) Experimental Environment, (b)Test for Mobile Device, (c) Test for Hololens

[Table 3] Evaluating Items for Usability and the Explanations

\begin{tabular}{cc}
\hline Classification & Description \\
\hline Awareness & GUI element and degree of awaring information \\
\hline Comfort & Comfort of eyes in the visual aspect \\
\hline Functionality & Structural stability or clarity of distinction \\
\hline Space Perception & Distinction (depth) between real and virtual objects \\
\hline
\end{tabular}

The survey of Park and Kim(2017) was used as a reference for conducting a user experience test [13]. The survey was composed of four items including awareness, comfort, functionality, and space perception. Subjects 
were asked to respond in five-point Likert scale method (i.e., very poor, poor, average, good, and very good).

\subsection{Data Processing}

Collected data were analyzed statistically by using IBM SPSS Statistics 24. Kolmogorov-Smirnov test was conducted to test the normality of the sample distribution $(\mathrm{p}>0.05)$. Therefore, Mann-Whitney test, a non-parametric test, was used to compare the two groups.

\section{Results and Analysis}

[Table. 4] Evaluation Results of User Experiences(A)

\begin{tabular}{|c|c|c|c|c|}
\hline Classification & Group (N) & $\begin{array}{c}\text { Awareness } \\
(\text { Mean } \pm \text { SD) }\end{array}$ & Z & p-value \\
\hline \multirow{2}{*}{ Awareness } & Mobile Device (22) & $4.40 \pm .66$ & \multirow{2}{*}{-1.655} & \multirow{2}{*}{.098} \\
\hline & HoloLens (22) & $3.86 \pm 1.08$ & & \\
\hline \multirow{2}{*}{ Comfort } & Mobile Device (22) & $3.59 \pm .79$ & \multirow{2}{*}{-.854} & \multirow{2}{*}{.393} \\
\hline & HoloLens (22) & $3.40 \pm .73$ & & \\
\hline \multirow{2}{*}{ Functionality } & Mobile Device (22) & $3.86 \pm .99$ & \multirow{2}{*}{-.437} & \multirow{2}{*}{.662} \\
\hline & HoloLens (22) & $3.72 \pm 1.03$ & & \\
\hline \multirow{2}{*}{$\begin{array}{c}\text { Space } \\
\text { Perception }\end{array}$} & Mobile Device (22) & $3.68 \pm .71$ & \multirow{2}{*}{-.447} & \multirow{2}{*}{.655} \\
\hline & HoloLens (22) & $3.86 \pm .83$ & & \\
\hline
\end{tabular}

The results of content A's user experience showed that awareness of the mobile device group (4.40土.66) was higher than that of the HoloLens group $(3.86 \pm 1.08)$ but they were not significantly different $(\mathrm{p}>0.05)$. The

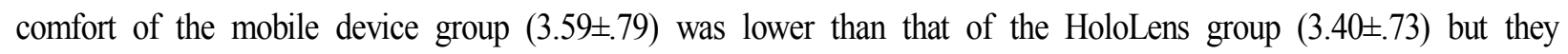

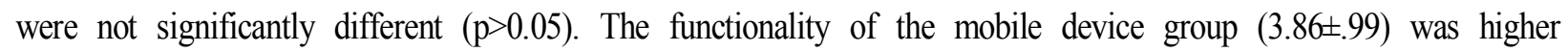
than that of the HoloLens group (3.72 \pm 1.03$)$ but they were not significantly different $(p>0.05)$. The space

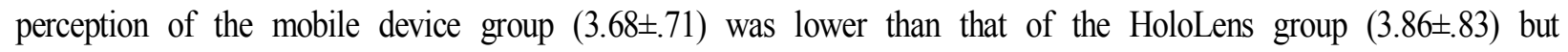
they were not significantly different $(\mathrm{p}>0.05)$.

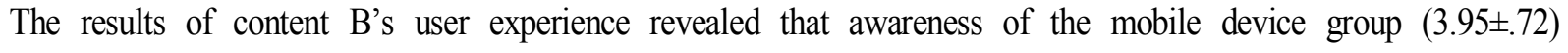
was higher than that of the HoloLens group $(3.86 \pm 1.08)$ but they were not significantly different $(\mathrm{p}>0.05)$. The

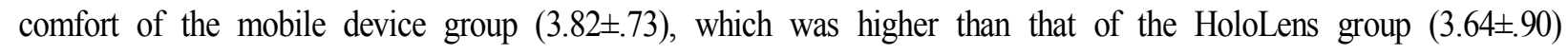

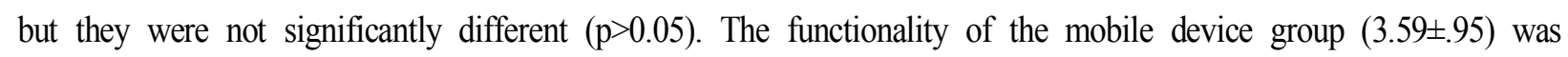

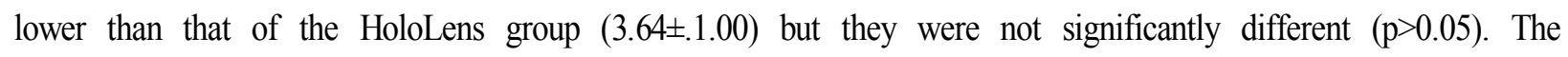

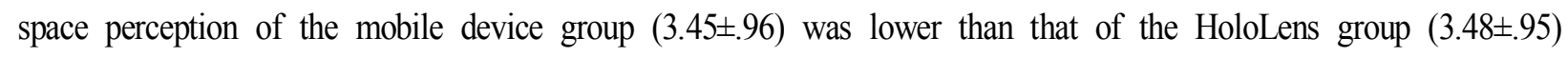
but they were not significantly different $(p>0.05)$. 
[Table 5] Evaluation Results of User Experiences(B)

\begin{tabular}{|c|c|c|c|c|}
\hline Classification & Group (N) & $\begin{array}{c}\text { Awareness } \\
(\text { Mean } \pm \mathrm{SD})\end{array}$ & Z & p-value \\
\hline \multirow{2}{*}{ Awareness } & Mobile Device (22) & $3.95 \pm .72$ & \multirow{2}{*}{-.025} & \multirow{2}{*}{.980} \\
\hline & HoloLens (22) & $3.86 \pm 1.08$ & & \\
\hline \multirow{2}{*}{ Comfort } & Mobile Device (22) & $3.82 \pm .73$ & \multirow{2}{*}{-.621} & \multirow{2}{*}{.535} \\
\hline & HoloLens (22) & $3.64 \pm .90$ & & \\
\hline \multirow{2}{*}{ Functionality } & Mobile Device (22) & $3.59 \pm .95$ & \multirow{2}{*}{-.200} & \multirow{2}{*}{.842} \\
\hline & HoloLens (22) & $3.64 \pm 1.00$ & & \\
\hline \multirow{2}{*}{$\begin{array}{c}\text { Space } \\
\text { Perception }\end{array}$} & Mobile Device (22) & $3.45 \pm .96$ & \multirow{2}{*}{-.941} & \multirow{2}{*}{.941} \\
\hline & HoloLens (22) & $3.48 \pm .95$ & & \\
\hline
\end{tabular}

\section{Conclusions and Future Studies}

This study is an initial study for evaluating the user experience required for developing augmented reality contents in the automobile maintenance situation. This study realized two contents and asked the subjects to observe them by using a mobile device and HoloLens. Afterward, user experience evaluation was conducted. No significant differences were found between the mobile device user experience and the HoloLens user experience for both contents. However, the awareness showed the largest difference between the mobile device group and the HoloLens group. It is believed that it was due to the limitation of the HoloLens hardware that is developed so far. On the other hand, the space perception of HoloLens was higher than that of the mobile device for content A and B. It was speculated that it was because HoloLens had a superior sense of immersion as an HMD device despite the difference in resolution and camera performance. Moreover, it is believed that the augmented reality contents used in this study presented the structure of the piston in an automobile engine miniature translucently so users concentrated on the structural information of the piston too much not to make differences in comfort and functionality.

Moreover, it is necessary to overcome the limitation of HoloLens hardware developed so far by preparing a visualization method considering various automobile maintenance situations. Particularly, this study confirmed that HoloLens has limitations in tracking an object in each frame and it is required to study how to match 3D model further to complement the limitations. Compared to the current mobile devices, the form of HoloLens is in the initial stage so it is predicted that it will offer better user experience as its performance will be improved in the future continuously. 


\section{References}

[1] Sung Ho Choi, Myoung Hun Lee, Jae Yeol Lee. "A Comparative Analysis of User Experience in Home Energy Saving Awareness Using Immersive Virtual Reality and Mobile Augmented Reality." Korean Journal of Computational Design and Engineering, 21.4 (2016): 397-408.

[2] Myeong-Hyeon Heo, Eun-Young Lee, Dongho Kim. "A Verification of the Effectiveness of Spatial Augmented Reality-based CCA for the Improvement of Traditional Sports Climbing Lessons." Journal of The Korea Contents Association, 17.5 (2017): 90-99.

[3] Soyoun Park, Viet Tran Hoang, Anh Nguyen Hoang, Gyohwa Bae, Jaewoon Lee, Dongho Kim. "Automobile Maintenance Training System using Phased Learning based on Virtual Reality." Journal of KIISE : Computing Practices and Letters, 19.12 (2013): 663-667.

[4] $\mathrm{Wu}$, Hsin-Kai, et al. "Current status, opportunities and challenges of augmented reality in education." Computers \& Education 62 (2013): 41-49.

[5] Martín-Gutiérrez, Jorge, et al. "Augmented reality to promote collaborative and autonomous learning in higher education." Computers in Human Behavior 51 (2015): 752-761.

[6] Milgram, Paul, and Fumio Kishino. "A taxonomy of mixed reality visual displays." IEICE TRANSACTIONS on Information and Systems 77.12 (1994): 1321-1329.

[7] Azuma, Ronald T. "A survey of augmented reality." Presence: Teleoperators and virtual environments 6.4 (1997): $355-385$.

[8] Billinghurst, Mark. "Augmented reality in education." New horizons for learning 12 (2002).

[9] Shinhyo Kim, So Young Han, Jusub Kim. "Hololens-based Immersive Interactive Storytelling Design : From "Sit \& Watch" to "Walk \& Experience." HCI Korea 2018, (2017): 425-428.

[10] Kim, Hee-young. "The Current Status and Development Direction of Mixed Reality Content." Catoon \& Animation Studies, 46 (2017.03): 181-206.

[11] https://unity3d.com/kr, Nov 1, (2017)

[12] https://www.vuforia.com, Nov 1, (2017)

[13] Park, Jin Hong, Kim, Hun. "Research on GUI(Graphic User Interface) Design Styles in Mixed Reality Service - Focusing on the Way to Distinguish between Reality and Virtual Object." Journal of The Korean Society Design Culture, 23.2 (2017): 271-282. 